\title{
Empatia em estudantes e profissionais de saúde: análise fatorial da Escala
}

\section{Multidimensional de Reatividade Interpessoal}

\author{
Empathy in health professionals and students: factorial analysis of the Brazilian version of \\ Interpersonal Reactivity Index \\ Empatía en estudiantes y profesionales de la salud: análisis factorial de la Escala Multidimensional \\ de Reactividad Interpersonal
}

Recebido: 10/02/2022 | Revisado: 16/02/2022 | Aceito: 20/02/2022 | Publicado: 02/03/2022

Carla Corradi-Perini
ORCID: https://orcid.org/0000-0002-9340-8704
Pontifícia Universidade Católica do Paraná, Brasil
E-mail: carla.corradi@ @ucpr.br
Ester Silvino da Costa Paris
ORCID: https://orcid.org/0000-0002-9180-6207
Pontifícia Universidade Católica do Paraná, Brasil
E-mail: esterparis@ @otmail.com
Marianna Rodrigues Beltrão
ORCID: https://orcid.org/0000-0003-2347-9109
Pontifícia Universidade Católica do Paraná, Brasil
E-mail: marianna.beltrao@ @otmail.com
Marisa Inês Corradi
ORCID: https://orcid.org/0000-0002-7854-779X
Pontifícia Universidade Católica do Paraná, Brasil
E-mail: m.corradi@pucpr.br
Raquel Martín-Martín
URCID: https://orcid.org/0000-0002-6022-7069
Universidad de Sevilla, Espanha
E-mail: raquel.martinm@sjd.es
José María Galán González-Serna
ORCID: https://orcid.org/0000-0002-5026-4333
Universidad de Sevilla, Espanha
E-mail: josemaria.galan@sjd.es

\section{Resumo}

Este estudo tem o objetivo de avaliar a empatia de estudantes e profissionais da área de saúde de Curitiba e verificar a estrutura fatorial da Escala Multidimensional de Reatividade Interpessoal nesta população. Participaram do estudo 886 indivíduos, do sexo feminino $(64,2 \%)$ e masculino, com idades de 18 a 65 anos (média=25,2 anos; desvio padrão=7,8), sendo 672 universitários e 214 profissionais, ambos da área da saúde da cidade de Curitiba, Paraná. O instrumento utilizado foi a Escala Multidimensional de Reatividade Interpessoal. Os estudantes apresentam maiores níveis de empatia global, angústia pessoal e fantasia que os profissionais. Os resultados relacionados às diferenças entre o sexo feminino e masculino não condizem com o que se apresenta na literatura nacional e internacional, o que pode ser atribuído a maior idade observada no sexo feminino. A escala apresenta adequada consistência interna e indicadores psicométricos aceitáveis para ser utilizada nessa população.

Palavras-chave: Empatia; Estudantes de ciências da saúde; Profissionais de saúde.

\begin{abstract}
This study aims to evaluate the empathy of health undergraduate students and professionals in Curitiba and to verify the factorial structure of the Brazilian version of Interpersonal Reactivity Index in this population. The participants were 886 individuals, female $(64.2 \%)$ and male, aged 18 to 65 years (mean $=25.2$ years; standard deviation $=7.8$ ), being 672 health undergraduate students and 214 health professionals. The instrument used was the Multidimensional Scale of Interpersonal Reactivity. Students have higher levels of global empathy, personal distress and fantasy than professionals. The results related to the differences between the female and male do not match what is presented in the national and international scientific literature, which can be attributed to the higher age observed in the women. Anyway, the scale has adequate internal consistency and acceptable psychometric indicators to be used in this population.
\end{abstract}

Keywords: Empathy; Health sciences students; Health professionals. 


\begin{abstract}
Resumen
Este estudio tiene como objetivo evaluar la empatía de estudiantes y profesionales del área de salud de Curitiba y verificar la estructura factorial de la Escala Multidimensional de Reactividad Interpersonal en esta población. Participaron del estudio un total de 886 individuos, mujeres (64,2\%) y hombres, con edades entre 18 y 65 años (media=25,2 años; desviación estándar=7,8), 672 estudiantes universitarios y 214 profesionales, ambos del área de la salud en el ciudad de Curitiba, Paraná. El instrumento utilizado fue la Escala Multidimensional de Reactividad Interpersonal. Los estudiantes muestran niveles más altos de empatía global, angustia personal y fantasía que los profesionales. Los resultados relacionados con las diferencias entre mujeres y hombres no son consistentes con lo presentado en la literatura nacional e internacional, lo que puede atribuirse a la mayor edad observada en las mujeres. La escala tiene una consistencia interna adecuada e indicadores psicométricos aceptables para ser utilizada en esta población.
\end{abstract}

Palabras clave: Empatía; Estudiantes de ciencias de la salud; Profesionales de la salud.

\title{
1. Introdução
}

A empatia dos profissionais de saúde está associada positivamente a uma maior satisfação do paciente, maior capacidade deste para o autocuidado, maior adesão ao tratamento, melhores resultados clínicos e redução da ansiedade e do sofrimento dos pacientes (Dambha-Miller et al., 2019; Derksen et al., 2013). Adicionalmente, os altos níveis de empatia se associam à realização pessoal do profissional (Bogiatzaki et al., 2019). Por outro lado, baixos níveis de empatia estão associados a altos níveis de burnout (Bogiatzaki et al., 2019; Ferri et al., 2015; Wilkinson et al., 2017).

Apesar da empatia ser um aspecto importante do bem-estar do paciente e dos profissionais, uma vasta literatura mostra uma escassez ou declínio da empatia nos profissionais e estudantes da área da saúde. Nos profissionais, tem se observado que a empatia diminui com a idade e a prática profissional (Ferri et al., 2015). Nos estudantes, o declínio da empatia ao longo da formação em saúde tem sido relacionada com aspectos curriculares formais e informais (ambientes de aprendizagem inadequados, modelos insatisfatórios, curta permanência hospitalar dos pacientes), currículo oculto (maus-tratos por parte dos superiores, vulnerabilidade dos estudantes frente aos supervisores, falta de apoio social, alta carga de trabalho), prática clínica (sendo o primeiro contato com os pacientes como uma fase de vulneração) e a angústia (Burnout, baixa qualidade de vida, depressão) (González-Serna et al., 2014; Mahoney et al., 2016; Neumann et al., 2011).

Nesse sentido, intervenções direcionadas ao desenvolvimento da empatia de estudantes e profissionais de saúde têm sido empregadas com resultados positivos no incremento da empatia (Bas-Sarmiento et al., 2017; Kelm et al., 2014).

Uma metanálise mostra que não existe consenso sobre quais instrumentos são mais confiáveis para avaliar a empatia clínica em estudantes da área da saúde (Fragkos et al., 2019). Os resultados dessa metanálise demonstram que confiabilidade geral das escalas é aceitável, mas houve heterogeneidade e viés de publicação. Fragkos et al. (2019) declaram que isso é explicado em parte pelas diferentes escalas e distintos países dos estudos e tipos de estudantes, justificando a necessidade de mais estudos que possam contribuir com futuras metanálises para consenso do uso de escalas, definições e componentes da empatia na área da saúde.

Diante da falta de consenso na definição de empatia (Fragkos et al., 2019), antes de avaliá-la é necessário entender a compreensão de empatia por parte dos autores que desenvolveram as escalas. A definição da empatia utilizada em nosso estudo compreende a capacidade de entender as emoções e/ou perspectivas dos outros e, frequentemente, ressoar com os estados emocionais dos outros. É uma resposta afetiva idêntica ou muito parecida com o que a outra pessoa está sentindo ou que se possa esperar, considerando o contexto, uma resposta derivada da compreensão do estado emocional ou condição de outra pessoa (Batson et al., 1991). Portanto, optou-se por utilizar o Índice de Reatividade Interpessoal de Davis (1980, 1983) que compreende a empatia em duas dimensões, incluindo empatia cognitiva e empatia emocional. Empatia cognitiva é a capacidade de entender as experiências, preocupações e perspectivas de outra pessoa, e empatia emocional é compartilhar a emoção de outra pessoa, envolvendo essencialmente o afeto pelo outro (Hall \& Schwartz, 2019; Smith, 2006).

No Brasil, o Índice de Reatividade Interpessoal (IRI) (Davis, 1980, 1983) foi adaptado e validado na estrutura 
tetrafatorial por Sampaio et al. (2011) com o nome de Escala Multidimensional de reatividade interpessoal (EMRI). A EMRI engloba as duas dimensões (cognitiva e emocional) da empatia, sendo uma medida da empatia disposicional que toma como ponto de partida a noção de que a empatia consiste em um conjunto de construções separadas, mas relacionadas. A partir dessa compreensão de empatia, o objetivo deste estudo foi avaliar a empatia de estudantes e profissionais da área de saúde de Curitiba-PR e verificar a estrutura fatorial da escala EMRI nesta população.

\section{Metodologia}

Trata-se de um estudo transversal com delineamento descritivo e abordagem quantitativa, aprovado pelo Comitê de Ética em Pesquisa da Pontifícia Universidade Católica do Paraná, sob parecer de número 3.271.123/2019.

\subsection{Participantes}

Participaram deste estudo, 886 indivíduos, do sexo feminino (64,2\%) e masculino (35,8\%), com idades de 18 a 65 anos (média=25,2 anos; desvio padrão=7,8; erro padrão da média=0,26), sendo 672 estudantes de graduação e 214 profissionais da área da saúde da cidade de Curitiba, Paraná. Foram incluídos estudantes de diferentes cursos da área da saúde (Medicina $=435 / 64,7 \%$ ～Enfermagem $=113 / 16,8 \% ; \quad$ Psicologia $=69 / 10,3 \% ; \quad$ Odontologia=27/4\%; Nutrição 19/2,8\%; Fisioterapia $=8 / 1,2 \%$; Biomedicina $=1 / 0,1 \%)$ e de diferentes anos $\left(1^{\circ}\right.$ ano $=109 / 16,2 \% ; 2^{\circ}$ ano $=191 / 28,4 \% ; 3^{\circ}$ ano $=119 / 17,7 \% ; 4^{\circ}$ ano=96/14,3\%; $5^{\circ}$ ano=103/15,3\%; $6^{\circ}$ ano=54/8\%). Os profissionais estavam distribuídos em: Enfermeiros (N=130/60,7\%); Médicos (N=22/10,3\%); Nutricionistas ( $\mathrm{N}=22 / 10,3 \%)$; Psicólogos (N=18/8,4\%); Fisioterapeutas (N=11/5,1\%); Farmacêuticos $(\mathrm{N}=4 ; 1,9 \%)$; Odontólogos, Terapeutas ocupacionais, Fonoaudiólogos e Assistentes sociais (representavam juntos menos de $3 \%$ do total).

\subsection{Instrumentos}

A EMRI é composta por 26 itens que descrevem o comportamento, sentimentos e características para avaliar dimensões da empatia (cognitiva e emocional) em quatro subescalas cada uma explorando uma faceta da empatia separadamente: angústia pessoal (AP; 6 itens), consideração empática (CE; 7 itens), tomada de perspectiva (TP; 6 itens) e fantasia (FS; 7 itens). A subescala AP avalia sentimentos afetivos de apreensão e desconforto direcionadas para o self em contextos interpessoais tensos, em que se imagina o sofrimento do outro. A subescala CE relaciona-se com a capacidade de experienciar sentimentos de compaixão e preocupação pelo outro, potencializando o desejo de ajudar pessoas em desvantagem, necessidade ou perigo. A subescala TP compreende a dimensão cognitiva do indivíduo de se colocar no lugar das outras pessoas, identificando pensamentos e sentimentos destas. A subescala $\mathrm{F}$ avalia a propensão da pessoa para se colocar em situações fictícias, a tendência de transpor a si mesmo imaginativamente (Davis, 1983).

Cada um dos itens da EMRI foram avaliados por escalas likert, variando de 1 (não me descreve bem) a 5 (descreveme muito bem). Os escores de cada uma das subescalas é a soma das pontuações dos respectivos itens que as compõem, sendo que o item 2, que integra a subescala FS deve ter a pontuação invertida. A soma dos escores de todas as subescalas é interpretada como o nível global de empatia.

\subsection{Procedimentos}

A coleta de dados foi realizada no período de maio a novembro de 2019. Profissionais e estudantes foram abordados pelos pesquisadores, em seus intervalos de trabalho e de aula, respectivamente, em três hospitais (um público e dois privados) e em uma universidade privada da cidade de Curitiba, e responderam à escala nesse momento ou em momento agendado, de forma voluntária e anônima. A participação foi condicionada ao aceite mediante assinatura do termo de consentimento livre e 
esclarecido.

\subsection{Análise de dados}

O programa estatístico IBM SPSS Statistics versão 20 foi utilizado para as estatísticas descritivas; teste de Kolmogorov-Smirnov para analisar normalidade das variáveis; teste U de Mann-Whitney para comparação das médias de empatia global e suas subescalas entre estudantes e profissionais e também por idade e sexo; teste de Spearman para correlação entre as subescalas da empatia; Alfa de Cronbach para análise da consistência interna e análise fatorial exploratória considerando que os valores de confiabilidade $\geq 0,70$ são adequados (Cronbach, 1951).

A validade do construto foi avaliada mediante a análise fatorial exploratória. O teste de esfericidade de Bartlett e a medida de adequação da amostra de Kaiser-Meyer-Olkin foram utilizados para verificar a idoneidade da análise fatorial. Foi realizada uma análise de componentes principais (ACP) com rotação varimax. Foram necessários valores próprios maiores que 1 para reter os fatores componentes, e cargas fatoriais de 0,30 ou mais foram consideradas satisfatórias para a interpretação da estrutura fatorial. As comunalidades foram calculadas para avaliar a estrutura do fator.

A análise fatorial confirmatória foi realizada com base na orientação metodológica e estatística dos estudos de Sampaio et al (2011) e de Formiga et al. (2013), utilizando-se o procedimento de Modelagem de Equações Estruturais, por meio do programa IBM SPSS Amos Graphics 24.0. O método de estimação de probabilidade máxima foi utilizado. A qualidade do modelo de medição foi verificada pelos seguintes índices de qualidade dos ajustes (Boomsma, 2000): i) razão entre o valor do qui-quadrado e os graus de liberdade $\left(\chi^{2} / \mathrm{gl}\right)$, cujo valor deve ser menor que 3 (Kline, 2005) e com limites de até 5 (Hair et al., 2006); ii) índices absolutos de melhor desempenho GFI (Goodness of Fit Index) (Jöreskog \& Sörbom, 1986) e AGFI (Adjusted Goodness-of-Fit Index) (Hoyle \& Panter, 1995) em intervalo entre 0 e 1 e aqueles que excedem 0,9 são considerados modelos adequados; iii) índice de ajuste comparativo (CFI), cujo valor deve ser igual ou superior a 0,90 ; iv) índice de Tucker-Lewis (TLI) que deve ser igual ou superior a 0,90 (Hair et al., 2006) Além disso, foi incluída a raiz do resíduo quadrático de aproximação média (RMSEA), que deve ser menor que 0,06 ou assumir um valor máximo de 0,08 , e a raiz do resíduo quadrático médio (RMR) que deve ser igual ou menor que 0,08 (Byrne, 2001).

\section{Resultados}

\subsection{Análise descritiva dos níveis de empatia global e suas dimensões}

Os escores do nível global de empatia e suas subescalas estão apresentados na tabela 1, com destaque para pontuações maiores para estudantes nas variáveis empatia global $(\mathrm{p}=0,02)$, angústia pessoal $(\mathrm{p}=0,002)$ e fantasia $(\mathrm{p}<0,001)$ quando comparados aos profissionais. Na comparação das médias segundo sexo, observou-se uma maior pontuação na subescala fantasia pelas mulheres $(\mathrm{p}=0,03)$. Os escores de empatia global $(\mathrm{r}=-0,070 ; \mathrm{p}=0,03)$, angústia pessoal $(\mathrm{r}=-0,079 ; \mathrm{p}=0,016)$ e fantasia $(r=-0,185 ; p<0,001)$ apresentaram uma correlação negativa muito fraca com a idade. Por outro lado, a subescala de tomada de perspectiva apresentou uma correlação positiva muito fraca com a idade $(r=0,110 ; p=0,001)$. Importante ressaltar que neste estudo as mulheres apresentam uma média de idade maior que os homens $(\mathrm{p}<0,001)$. 
Tabela 1. Escores do nível global de empatia e suas dimensões a partir da aplicação da Escala Multidimensional de Reatividade Interpessoal em estudantes de graduação e profissionais da área da saúde. Curitiba, Paraná, Brasil, 2019.

\begin{tabular}{|c|c|c|c|c|c|}
\hline EMRI & Mínimo & Máximo & Média & Erro padrão da média & Desvio padrão \\
\hline \multicolumn{6}{|l|}{ Empatia global* } \\
\hline Amostra $1^{\mathrm{a}}$ & 41 & 126 & 92,8 & 0,47 & 13,9 \\
\hline Amostra $2^{b}$ & 50 & 122 & 93,6 & 0,52 & 13,5 \\
\hline Amostra $3^{c}$ & 41 & 126 & 90,3 & 1,00 & 14,7 \\
\hline \multicolumn{6}{|l|}{ Angústia pessoal** } \\
\hline Amostra $1^{\mathrm{a}}$ & 6 & 30 & 17,0 & 0,17 & 5,1 \\
\hline Amostra $2^{\mathrm{b}}$ & 6 & 30 & 17,3 & 0,19 & 4,5 \\
\hline Amostra $3^{c}$ & 6 & 30 & 16,1 & 0,39 & 5,7 \\
\hline \multicolumn{6}{|l|}{ Consideração empática } \\
\hline Amostra $1^{\mathrm{a}}$ & 11 & 35 & 28,1 & 0,15 & 4,5 \\
\hline Amostra $2^{\mathrm{b}}$ & 12 & 35 & 28,2 & 0,18 & 4,5 \\
\hline Amostra $3^{c}$ & 11 & 35 & 28,1 & 0,31 & 4,5 \\
\hline \multicolumn{6}{|l|}{ Tomada de perspectiva } \\
\hline Amostra $1^{\mathrm{a}}$ & 8 & 30 & 23,6 & 0,12 & 3,5 \\
\hline Amostra $2^{\mathrm{b}}$ & 8 & 30 & 23,5 & 0,13 & 3,5 \\
\hline Amostra $3^{c}$ & 11 & 30 & 24,0 & 0,24 & 3,4 \\
\hline \multicolumn{6}{|l|}{ Fantasia*** } \\
\hline Amostra $1^{\mathrm{a}}$ & 7 & 35 & 24,1 & 0,19 & 5,7 \\
\hline Amostra $2^{b}$ & 7 & 35 & 24,7 & 0,22 & 5,7 \\
\hline Amostra $3^{c}$ & 7 & 35 & 22,3 & 0,38 & 5,6 \\
\hline
\end{tabular}

EMRI: Escala Multidimensional de Reatividade Interpessoal; $a=$ amostra total $(\mathrm{N}=886)$; b=estudantes de graduação da área da saúde $(\mathrm{N}=672) ; \mathrm{c}=$ profissionais da área da saúde $(\mathrm{N}=214)$. Teste $\mathrm{U}$ de Mann-Whitney: *diferença significativa ao nível de 0,05 ; **diferença significativa ao nível de 0,$01 ; * * *$ diferença significativa ao nível de 0,001 entre estudantes e profissionais da área da saúde. Fonte: Autores.

\subsection{Confiabilidade e análise fatorial exploratória}

A análise da confiabilidade da escala e de suas dimensões, por meio do Alfa de Cronbach, permitiu comprovar que apresentam índices adequados de consistência interna (Tabela 2). O teste de Adequação da Amostra Kaiser-Meyer-Olkin (KMO) resultou em um valor de 0,901 e o teste de esfericidade de Barlett mostrou-se significante ( $<<0,001)$, o que indica que o método da Análise Fatorial é adequado para o tratamento dos dados. A análise de componentes principais indicou que os 26 itens da EMRI poderiam ser compreendidos a partir de um modelo com cinco fatores, os quais explicariam cerca de 54,42\% da variância observada. Como o objetivo era avaliar a adequação do modelo proposto por Sampaio et al. (2011) aos estudantes de graduação e profissionais da área da saúde, as análises foram realizadas com o modelo tetrafatorial, cujos parâmetros da análise fatorial exploratória (pelo método de extração de componentes principais e de rotação varimax) estão demonstradas na Tabela 2 (valores inferiores a 0,3 foram eliminados para facilitar a leitura). 
Tabela 2. Análise fatorial exploratória da Escala Multidimensional de Reatividade Interpessoal. Matriz de configuração. Pesos fatoriais, variância explicada e Alfa de Cronbach ( $\mathrm{N}=886$ estudantes de graduação e profissionais da área da saúde). Curitiba, Paraná, Brasil, 2019.

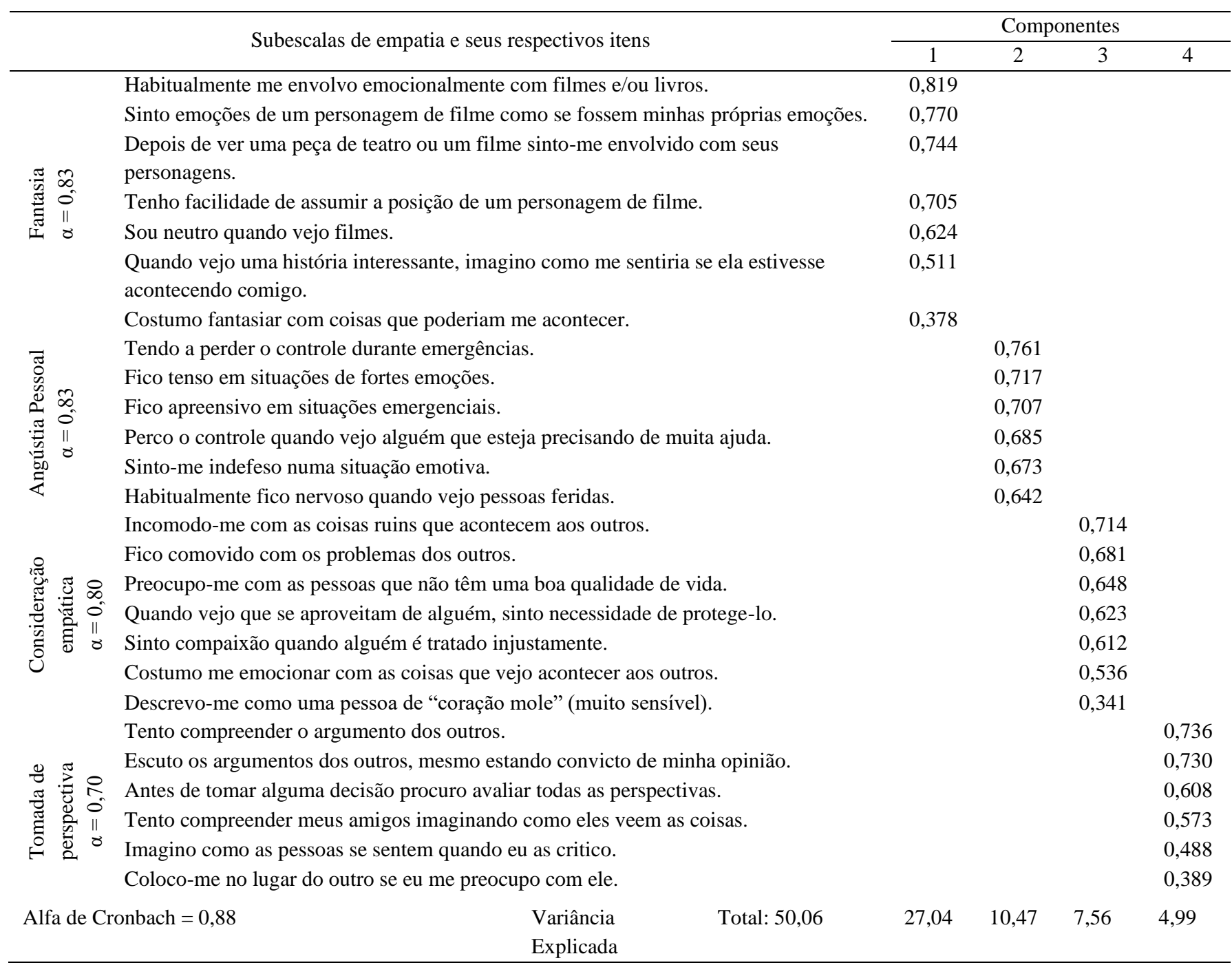

Método de extração: Análise de componentes principais. Método de rotação: Varimax com Normalização Kaiser. Rotação convergiu em 5 interações. Fonte: Autores.

Todas as dimensões da EMRI se correlacionaram positivamente entre si ao nível de p<0,001 (Tabela 3). Quando analisados separadamente estudantes e profissionais, e na análise segundo o sexo, as correlações se mantiveram no mesmo nível (p<0,001), com exceção das dimensões TP e AP, cuja correlação (positiva) foi de 0,002 nos homens.

Tabela 3. Correlações entre as dimensões da Escala Multidimensional de Reatividade Interpessoal (EMRI) (N=886 estudantes de graduação e profissionais da área da saúde). Curitiba, Paraná, Brasil, 2019.

\begin{tabular}{lllll}
\hline Subescalas de empatia & EMRI & CE & FS & AP \\
\hline Consideração empática (CE) & 0,814 & & & \\
Fantasia (FS) & 0,772 & 0,522 & & \\
Angústia pessoal (AP) & 0,715 & 0,453 & 0,391 & \\
Tomada de perspectiva (TP) & 0,521 & 0,436 & 0,216 & 0,140 \\
\hline
\end{tabular}

Todas as correlações foram significativas ao nível de 0,001 (Correlação de Spearman, bilateral). Fonte: Autores. 


\subsection{Análise fatorial confirmatória}

A análise fatorial confirmatória objetivou testar a adequação do modelo tetrafatorial nas amostras de estudantes e profissionais da área de saúde e apresenta os indicadores psicométricos da estrutura fatorial da EMRI próximos aos recomendados pela literatura, tanto para a amostra total, quanto para os estudantes e profissionais, separadamente (Tabela 4).

Tabela 4. Indicadores psicométricos da estrutura fatorial da Escala Multidimensional de Reatividade Interpessoal. ( $N=886$ estudantes de graduação e profissionais da área da saúde). Curitiba, Paraná, Brasil, 2019.

\begin{tabular}{lccccccc}
\hline Amostras & $\mathrm{x}^{2} / \mathrm{gl}$ & RMR $^{*}$ & GFI & AGFI & CFI & TLI & RMSEA \\
\hline Amostra $1^{\mathrm{a}}$ & 3,461 & 0,056 & 0,926 & 0,901 & 0,918 & 0,898 & 0,053 \\
Amostra 2 $^{\mathrm{b}}$ & 2,965 & 0,063 & 0,915 & 0,888 & 0,907 & 0,887 & 0,054 \\
Amostra 3 $^{\mathrm{c}}$ & 1,599 & 0,066 & 0,865 & 0,832 & 0,921 & 0,908 & 0,053
\end{tabular}

$\mathrm{a}=$ amostra total $(\mathrm{N}=886) ; \mathrm{b}=$ estudantes de graduação da área da saúde $(\mathrm{N}=672)$; $=$ profissionais da área da saúde $(\mathrm{N}=214) ; * \mathrm{RMR}$ padronizado; RMR: Raiz Quadrada Media Residual; GFI: Goodness-of-Fit Index; AGFI: Adjusted Goodness-of-Fit Index; CFI: Comparative Fit Index; TLI: Tucker-Lewis Index; RMSEA: Root-Mean-Square Error of Approximation; CAIC: Consistent Akaike Information Criterion; ECVI: Expected Cross-Validation Index. Fonte: Autores.

\section{Discussão}

O IRI, instrumento para avaliação de empatia proposto por Davis (1980), foi traduzido e validado para os idiomas e tem sido amplamente utilizado em diferentes populações. Apesar de não ter sido desenvolvida, especificamente, para estudantes e profissionais da saúde e ter sua validade questionada para esta população (Hong \& Han, 2020) é uma escala frequentemente utilizada para avaliar empatia na área da saúde, tanto em estudantes (Fragkos et al., 2019), quanto em profissionais (Hong \& Han, 2020).

A característica mais relevante do instrumento de Davis (1980) é que ele permite medir tanto o aspecto cognitivo quanto o emocional do indivíduo ao adotar uma atitude empática e por isso foi selecionado para este estudo. A dimensão cognitiva é avaliada com as subescalas de tomada de perspectiva e de fantasia. A dimensão emocional é acessada a partir das subescalas de angústia pessoal e de consideração empática (Davis, 1983).

Deve-se levar em conta que o IRI, mede a empatia disposicional ou baseada em traços, ou seja, avalia as tendências crônicas das pessoas em ter empatia, em qualquer situação relacional. Por outro lado, o IRI não seria apropriado para pesquisas sobre empatia situacional, que envolve respostas emocionais imediatas às situações de outros (Batson et al., 1987). Portanto, a partir do entendimento de que a empatia é um atributo do indivíduo importante para todas as relações interpessoais, e isso inclui a relação profissional-paciente, o IRI seria um instrumento adequado para avaliar a empatia na área da saúde. A questão que emerge é se o modelo é aplicável à área da saúde no Brasil.

O IRI foi adaptado e validado a primeira vez no Brasil por Koller et al. (2001) que retiraram os itens referentes à subescala fantasia e designaram o instrumento como Escala Multidimensional de Reatividade Interpessoal (EMRI). Uma década depois, o modelo tetrafatorial de Davis (1980, 1983), incluindo a subescala fantasia, é validado no Brasil por Sampaio et al. (2011). No primeiro processo de validação foram envolvidos adolescentes de 14 a 16 anos (Koller et al., 2001) e no segundo, estudantes universitários (incluindo da área da saúde) Sampaio et al. (2011). Entretanto, não encontramos até o momento um estudo com a análise confirmatória do modelo IRI validado no Brasil, especificamente, para estudantes e profissionais da área da saúde, sendo este o propósito deste estudo.

Ao realizar a análise fatorial confirmatória na amostra total (estudantes e profissionais), o modelo atendeu aos critérios exigidos para apoiar seu ajuste nos dados observados. Embora os valores aceitáveis sejam identificados em RMR $(0,056)$, GFI $(0,926)$, AGFI $(0,901)$, CFI $(0,918)$, TLI $(0,889)$ e RMSEA $(0,053)$, os valores da razão $\chi^{2} / \mathrm{gl}(3,461)$ estão no 
limite do valor recomendado (preferencialmente menor que 3, mas aceitável até 5). Para interpretar a razão $\chi^{2} / \mathrm{gl}$, devemos levar em consideração a falta de normalidade das variáveis deste estudo e o grande tamanho amostral $(\mathrm{N}=886)$, fatores que podem influenciar na interpretação do resultado, portanto, descartamos o resultado obtido na razão $\chi^{2} / \mathrm{gl}$ e contamos com o restante dos índices para afirmar a bondade do ajuste. Para confirmar tal inferência, realizamos a análise fatorial confirmatória nas amostras de estudantes e de profissionais separadamente. Pode-se observar que quando se trata de amostras mais homogêneas como no caso de profissionais o ajuste do modelo apresenta melhores resultados em relação a $\chi^{2} / \mathrm{gl}(1,599)$. Em relação aos estudantes, como estes eram de diferentes níveis de formação (do $1^{\circ}$. ao $6^{\circ}$. ano) e de diferentes cursos, o resultado obtido na razão $\chi^{2} / \mathrm{gl}$ apresentou-se no limite do entendido como adequado $(2,965)$. De fato, os níveis de empatia diferem entre os diferentes níveis de formação e diferentes cursos da área da saúde (Petrucci et al., 2016).

Assim, a partir dos resultados de consistência interna medidos pelo Alfa de Cronbach e o ajuste do modelo aos nossos dados a partir dos os indicadores psicométricos da estrutural fatorial, podemos afirmar que a versão traduzida e adaptada do IRI por Sampaio et al. (2011) é adequada para avaliação da empatia nos estudantes e profissionais da área da saúde de Curitiba.

Ao aplicar a escala em nossa amostra, pode-se observar que o nível global de empatia e os escores das subescalas de angústia pessoal e fantasia são significativamente maiores nos estudantes quando comparados aos profissionais. Um fator que pode explicar parcialmente essa diferença é a idade, que é significativamente maior nos profissionais $(\mathrm{p}<0,001)$. Além disso, nos testes de correlação de Spearman, foi encontrada uma correlação negativa (ainda que muito baixa, porém significativa) entre a idade e empatia global, angústia pessoal e fantasia. Ferri et al. (2015) observaram que a idade e prática profissional se relacionavam negativamente com os níveis de empatia. Especificamente, em relação à fantasia e angústia pessoal, o estudo de Fernández-Abascal e Martín-Díaz (2019). apresentou relações negativas significativas entre os escores dessas subescalas e a idade.

Apesar dessas diferenças, percebe-se que as pontuações nas subescalas apresentam um padrão semelhante nos estudantes e profissionais. Proporcionalmente ao escore máximo possível, percebe-se que ambas as amostras pontuaram mais na subescala CE, seguida da TP, FS e AP. Esse mesmo padrão foi observado no estudo de Sampaio et al. (2011) que incluiu estudantes universitários brasileiros. As subescalas de CE e TP são os componentes mais importantes da empatia, e conjuntamente, são responsáveis por uma resposta empática mais madura baseada na compreensão do outro com ressonância ao seu estado emocional (Mestre Escrivá et al., 2004).

Essa característica empática é esperada em estudantes e profissionais da saúde, pois, conforme a teoria de Davis (1983), maior expressão de TP reflete a capacidade de reconhecer as necessidades do outro e CE exprime a preocupação por aqueles que apresentam-se vulnerados, potencializando o desejo de buscar meios para aliviar o sofrimento do próximo. O estudo de Yu et al. (2019) demonstrou que pontuações mais altas em tomada de perspectiva e consideração empática demonstraram níveis mais altos de percepção em relação à necessidade do outro, justificando a importância da empatia nas relações profissional-paciente.

Interessantemente, com exceção da subescala fantasia, em nosso estudo não foram observados maiores níveis de empatia nas mulheres, como observados em outros estudos no Brasil e no mundo, utilizando a escala IRI. Não existem evidências em nossos dados que justifiquem esse resultado. Assim, sugere-se o desenvolvimento de novos estudos envolvendo outras instituições de ensino e de saúde na cidade de Curitiba, incluindo estudos qualitativos que possam compreender possíveis fatores antropológicos e culturais que justifiquem nossos achados (Atkins et al., 2016; Chopik et al., 2017; Formiga, 2016).

Outro dado interessante que diverge dos achados e teoria de Davis (1983), mas corrobora com outros estudos brasileiros incluindo o de Sampaio et al. (2011), é que a angústia pessoal se correlacionou positivamente com as outras subescalas da EMRI (Tabela 3). Sampaio et al. (2011) explicam que a angústia pessoal pode ativar a consideração empática, 
mobilizando um comportamento de ajuda em uma situação de sofrimento do outro, desde que a tomada de perspectiva também fosse elevada, fazendo com que o indivíduo (em nosso estudo podemos entender como estudante ou profissional de saúde) compreenda o que o outro (paciente) está sentindo. Muitas vezes, na área da saúde, essa compreensão envolve a capacidade de ler e interpretar corretamente os sinais não verbais em outros (Riggio \& Darioly, 2015).

O interesse pelo estudo da empatia na área da saúde se justifica pelos achados relevantes que a correlacionam positivamente com uma boa relação profissional-paciente. Considera-se a empatia como um atributo necessário para que o profissional compreenda a situação, perspectivas e sentimentos do paciente; para que se comunique de maneira efetiva; e para que sua atuação seja centrada na pessoa (Derksen et al., 2013; Finset \& Ørnes, 2017). Por outro lado, altos níveis de empatia parecem proteger do desenvolvimento de burnout, cada vez mais comum em estudantes e profissionais da área da saúde (Ferri et al., 2015; Marilaf Caro et al., 2017; Wilkinson et al., 2017). No entanto, quando a pessoa está sobrecarregada emocionalmente ou esgotada no aspecto profissional, a capacidade de empatia diminui como resultado desse esgotamento (Riess, 2017).

Portanto, considerando que a empatia pode ser desenvolvida durante a formação profissional em saúde, utilizando-se diferentes formas de intervenção (Gholamzadeh et al., 2018; Patel et al., 2019; ter Beest et al., 2018; van Berkhout \& Malouff, 2016), sugere-se a utilização da EMRI para avaliar essas intervenções (antes e após) nos estudantes e profissionais de saúde da cidade de Curitiba, tanto na graduação quanto na educação continuada, interpretando cuidadosamente os resultados obtidos em cada subescala. Isto fornecerá subsídios aos agentes de formação direcionarem as estratégias mais adequadas para cada profissão, com o objetivo fim de melhorar a qualidade da assistência em saúde e também proteger os estudantes e profissionais do potencial desgaste emocional resultante das relações interpessoais em saúde (Riess, 2017).

Este estudo apresenta algumas limitações que podem influenciar na interpretação dos resultados. Uma das limitações deste estudo é que, apesar da amostra ser relativamente grande, não apresenta homogeneidade no número de participantes dos diferentes cursos e profissões envolvidos, assim como a distribuição dos participantes em relação ao sexo e idade nos diferentes subgrupos é heterogênea. Ademais, é importante ressaltar que para a análise da empatia nos estudantes, envolveu-se uma única instituição de ensino superior, o que pode comprometer a generalização.

\section{Conclusão}

A escala EMRI apresenta adequada consistência interna e indicadores psicométricos da estrutura fatorial aceitáveis para ser utilizada para avaliar a empatia em estudantes e profissionais da área de saúde da cidade de Curitiba. Entretanto, os resultados relacionados às diferenças entre o sexo feminino e masculino não condizem com o que se apresenta na literatura nacional e internacional. Sugere-se a realização de estudos qualitativos com métodos das ciências sociais que possam proporcionar evidências que direcionem as estratégias de formação em saúde com foco na empatia.

\section{Referências}

Atkins, D., Uskul, A. K., \& Cooper, N. R. (2016). Culture shapes empathic responses to physical and social pain. Emotion, 16(5), 587-601. https://doi.org/10.1037/emo0000162

Bas-Sarmiento, P., Fernández-Gutiérrez, M., Baena-Baños, M., \& Romero-Sánchez, J. M. (2017). Efficacy of empathy training in nursing students: A quasiexperimental study. Nurse Education Today, 59, 59-65. https://doi.org/10.1016/j.nedt.2017.08.012

Batson, C. D., Fultz, J., \& Schoenrade, P. A. (1987). Adults' emotional reactions to the distress of others. In N. Eisenberg \& J. Strayer (Eds.), Cambridge studies in social and emotional development. Empathy and its development. (pp. 163-184). Cambridge University Press.

Batson, C. D., Batson, J. G., Slingsby, J. K., Harrell, K. L., Peekna, H. M., \& Todd, R. M. (1991). Empathic joy and the empathy-altruism hypothesis. Journal of Personality and Social Psychology, 61(3), 413-426. https://doi.org/10.1037/0022-3514.61.3.413 
Bogiatzaki, V., Frengidou, E., Savakis, E., Trigoni, M., Galanis, P., \& Anagnostopoulos, F. (2019). Empathy and Burnout of Healthcare Professionals in Public Hospitals of Greece International Journal of Caring http://search.ebscohost.com/login.aspx?direct=true \&db=cin20\&AN=138636049\&site=ehost-live

Boomsma, A. (2000). Reporting analyses of covariance structures. Structural Equation Modeling. https://doi.org/10.1207/S15328007SEM0703_6

Byrne, B. M. (2001). Structural equation modeling with AMOS: Basic concepts, applications, and programming. Mahwah, NJ: Lawrence Erlbaum Associates, Inc. https://doi.org/10.4324/9781410600219

Chopik, W. J., O’Brien, E., \& Konrath, S. H. (2017). Differences in Empathic Concern and Perspective Taking Across 63 Countries. Journal of CrossCultural Psychology, 48(1), 23-38. https://doi.org/10.1177/0022022116673910

Cronbach, L. J. (1951). Coefficient alpha and the internal structure of tests. Psychometrika, 16(3), 297-334. https://doi.org/10.1007/BF02310555

Dambha-Miller, H., Feldman, A. L., Kinmonth, A. L., \& Griffin, S. J. (2019). Association between primary care practitioner empathy and risk of cardiovascular events and all-cause mortality among patients with type 2 diabetes: A population- based prospective cohort study. Annals of Family Medicine, 17(4), 311-318. https://doi.org/10.1370/afm.2421

Davis, M. H. (1980). A Multidimensional Approach to Individual Differences in Empathy. JSAS Catalog of Selected Documents in Psychology, 10, 85. https://doi.org/10.1037/0022-3514.44.1.113

Davis, M. H. (1983). Measuring individual differences in empathy: Evidence for a multidimensional approach. Journal of Personality and Social Psychology, 44(1), 113-126. https://doi.org/10.1037/0022-3514.44.1.113

Derksen, F., Bensing, J., \& Lagro-Janssen, A. (2013). Effectiveness of empathy in general practice: A systematic review. British Journal of General Practice, 63(606), 76-84. https://doi.org/10.3399/bjgp13X660814

Fernández-Abascal, E. G., \& Martín-Díaz, M. D. (2019). Relations between dimensions of emotional intelligence, specific aspects of empathy, and non-verbal sensitivity. Frontiers in Psychology, 10(MAY). https://doi.org/10.3389/fpsyg.2019.01066

Ferri, P., Guerra, E., Marcheselli, L., Cunico, L., \& di Lorenzo, R. (2015). Empathy and burnout: An analytic cross-sectional study among nurses and nursing students. Acta Biomedica, 86(November), 104-115.

Finset, A., \& Ørnes, K. (2017). Empathy in the Clinician-Patient Relationship. Journal of Patient Experience, 4(2), 64-68. https://doi.org/10.1177/2374373517699271

Formiga, N. S., Rocha, M. C. O., Pinto, A. D. S. S., Reis, D. A. Dos, Costa, S. M. D. S., \& Leime, J. (2013). Fidedignidade da estrutura fatorial da Escala Multidimensional de Reatividade Interpessoal (EMRI). Estudos Interdisciplinares Em Psicologia, 4(1), 64. https://doi.org/10.5433/2236-6407.2013v4n1p64

Formiga, N. S. (2016). Verificação de um modelo teórico entre a empatia, socialização ética e orientação cultural em jovens brasileiros. Actualidades En Psicología, 30(120), 99. https://doi.org/10.15517/ap.v30i120.18156

Fragkos, K. C., Sotiropoulos, I., \& Frangos, C. C. (2019). Empathy assessment in healthcare students is highly heterogeneous: A systematic review and metaanalysis (2012-2016). World Journal of Meta-Analysis (Vol. 7). https://doi.org/10.13105/wjma.v7.i1.1

Gholamzadeh, S., Khastavaneh, M., Khademian, Z., \& Ghadakpour, S. (2018). The effects of empathy skills training on nursing students' empathy and attitudes toward elderly people. BMC Medical Education, 18(1), 1-7. https://doi.org/10.1186/s12909-018-1297-9

González-Serna, J. M. G., Serrano, R. R., Martín, M. S. M., \& Fernández, J. M. A. (2014). Descenso de empatía en estudiantes de enfermería y análisis de posibles factores implicados. Psicologia Educativa, 20(1), 53-60. https://doi.org/10.1016/j.pse.2014.05.007

Hair, J. F., Black, W. C., Babin, B., Anderson, R. E., \& Tatham, R. L. (2006). Multivariate Data Analysis (6th ed.). Upper Saddle River, NJ: Prentice-Hall.

Hall, J. A., \& Schwartz, R. (2019). Empathy present and future. The Journal of Social Psychology, 159(3), 225-243. https://doi.org/10.1080/00224545.2018.1477442

Hong, H., \& Han, A. (2020). A systematic review on empathy measurement tools for care professionals. Educational Gerontology, 46(2), 72-83. https://doi.org/10.1080/03601277.2020.1712058

Hoyle, R. H., \& Panter, A. T. (1995). Writing about structural equation models. In Structural equation Modelling: Concepts, Issues and Applications (pp. 159176). Thousand Oaks, CA: Sage.

Jöreskog, K. G., \& Sörbom, D. (1986). LISREL VI: Analysis of linear structural relationships by maximum likelihood and least square methods. Mooresville.

Kelm, Z., Womer, J., Walter, J. K., \& Feudtner, C. (2014). Interventions to cultivate physician empathy: A systematic review. BMC Medical Education, 14(1), 1-11. https://doi.org/10.1186/1472-6920-14-219

Kline, R. B. (2005). Principles and practice of structural equation modeling (2nd ed.). The Guilford Press.

Koller, S. H., Camino, C., \& Ribeiro, J. (2001). Adaptação e validação interna de duas escalas de empatia para uso no Brasil. Estudos de Psicologia, 18(3), 43-53.

Marilaf Caro, M., San-Martín, M., Delgado-Bolton, R., \& Vivanco, L. (2017). Empatía, soledad, desgaste y satisfacción personal en Enfermeras de cuidados paliativos y atención domiciliaria de Chile. Enfermería Clínica, 27(6), 379-386. https://doi.org/10.1016/j.enfcli.2017.04.007

Mahoney, S., Sladek, R. M., \& Neild, T. (2016). A longitudinal study of empathy in pre-clinical and clinical medical students and clinical supervisors. BMC Medical Education, 16(1), 1-8. https://doi.org/10.1186/s12909-016-0777-z 
Research, Society and Development, v. 11, n. 3, e42511326816, 2022

(CC BY 4.0) | ISSN 2525-3409 | DOI: http://dx.doi.org/10.33448/rsd-v11i3.26816

Mestre Escrivá, V., Frías Navarro, M. D., \& Samper García, P. (2004). La medida de la empatía: análisis del Interpersonal Reactivity Index. Psicothema, $16(2), 255-260$

Neumann, M., Edelhäuser, F., Tauschel, D., Fischer, M. R., Wirtz, M., Woopen, C., \& Scheffer, C. (2011). Empathy decline and its reasons: A systematic review of studies with medical students and residents. Academic Medicine, 86(8), 996-1009. https://doi.org/10.1097/ACM.0b013e318221e615

Patel, S., Pelletier-Bui, A., Smith, S., Roberts, M. B., Kilgannon, H., Trzeciak, S., \& Roberts, B. W. (2019). Curricula for empathy and compassion training in medical education: A systematic review. PLoS ONE, 14(8), 1-25. https://doi.org/10.1371/journal.pone.0221412

Petrucci, C., La Cerra, C., Aloisio, F., Montanari, P., \& Lancia, L. (2016). Empathy in health professional students: A comparative cross-sectional study. Nurse Education Today. https://doi.org/10.1016/j.nedt.2016.03.022

Riess, H. (2017). The Science of Empathy. Journal of Patient Experience, 4(2), 74-77. https://doi.org/10.1177/2374373517699267

Riggio, R. E., \& Darioly, A. (2015). Measuring nonverbal sensitivity. In APA handbook of nonverbal communication. https://doi.org/10.1037/14669-023

Sampaio, L. R., Guimarães, P. R. B., Camino, C. P. dos S., Formiga, N. S., \& Menezes, I. G. (2011). Estudos sobre a dimensionalidade da empatia tradução e adaptação do Interpersonal Reactivity Index (IRI). Psico, 42(1), 67-76.

Smith, A. (2006). Cognitive empathy and emocional empathy in human behavior and evolution. The Psychological Record, 56, 3-21.

ter Beest, H., van Bemmel, M., \& Adriaansen, M. (2018). Nursing student as patient: experiential learning in a hospital simulation to improve empathy of nursing students. Scandinavian Journal of Caring Sciences, 32(4), 1390-1397. https://doi.org/10.1111/scs.12584

van Berkhout, E. T., \& Malouff, J. M. (2016). The efficacy of empathy training: A meta-analysis of randomized controlled trials. Journal of Counseling Psychology, 63(1), 32-41. https://doi.org/10.1037/cou0000093

Wilkinson, H., Whittington, R., Perry, L., \& Eames, C. (2017). Examining the relationship between burnout and empathy in healthcare professionals: A systematic review. Burnout Research, 6(June), 18-29. https://doi.org/10.1016/j.burn.2017.06.003

Yu, J., Lee, S., Kim, M., Lim, K., Chang, K., \& Lee, M. (2019). Relationships Between Perspective-Taking, Empathic Concern, and Self-rating of Empathy as a Physician Among Medical Students. Academic Psychiatry, 1-4. https://doi.org/10.1007/s40596-019-01114-x 